\title{
Project AstroSat: five years of operations and continuing
}

\author{
S. Seetha ${ }^{1}$, V. Girish ${ }^{2, *}$ and V. Koteswara Rao ${ }^{3}$ \\ ${ }^{1}$ Raman Research Institute, Bengaluru 560 080, India \\ ${ }^{2}$ ISRO Headquarters, Bengaluru 560 231, India \\ ${ }^{3}$ U.R. Rao Satellite Centre, Bengaluru 560 017, India
}

India's first dedicated multi-wavelength satellite, AstroSat, was launched by PSLV C30 from the Satish Dhawan Space Centre, Sriharikota, Andhra Pradesh on 28 September 2015. It is India's first multiwavelength observatory. AstroSat carries five scientific payloads and is capable of simultaneous observations from ultraviolet to very hard $X$-rays. It has completed five years of on-board operations in September 2020 as a proposal-based observatory. Currently, it has close to 1500 global users and has resulted in more than 150 articles in peer-reviewed journals. This article is an overview providing a brief description of the AstroSat mission and some recent results using data from this unique Indian space observatory.

Keywords: Multi-wavelength satellite, proposal-based space observatory, scientific payloads, ultraviolet and $\mathrm{X}$-ray astronomy.

WITH the arrival of the space age in the 1950 s, astronomical observations at wavelengths shorter than the optical had opened up. Ultraviolet (UV), X-ray and gamma-ray studies using dedicated satellites contributed significantly to the understanding of the Universe. This has expanded in the late 1990s with the launch of several missions with improved sensitivity, and both image and spectral resolution. Around the same time, the success of science payloads on the SROSS series of satellites ${ }^{1}$ and the piggyback experiments on the IRS-P3 mission ${ }^{2}$ motivated the scientific community in India to propose to the Indian Space Research Organisation (ISRO) for a full-fledged astronomy mission. Though the four Great Observatory programmes of National Aeronautics and Space Administration (NASA), USA (Hubble Space Telescope (HST), Compton Gamma Ray Observatory, Chandra X-ray Observatory and Spitzer Space Telescope) which began in 1990 with the launch of HST, and European Space Agency's XMM-Newton revolutionized astronomy in different wavelength regions from infrared (IR) to gamma rays, there was a growing need for simultaneous multiwavelength studies of cosmic sources to directly reveal astrophysical processes that power the observed broadband emission, and how these evolve in time and space. It

\footnotetext{
*For correspondence. (e-mail: girishv@isro.gov.in)
}

is in this background, that the Indian astronomical community, after much debate, converged on the definition of a multi-wavelength satellite that covered the far and near UV and a wide range of X-ray bands of the electromagnetic spectrum simultaneously. Based on the proposal for experiments from the scientific community, on 3 April 2003, ISRO initiated India's first dedicated astronomy satellite project, AstroSat ${ }^{3}$.

\section{Scientific focus of AstroSat}

Detailed listing of the science objectives and instrument specifications with which the project was initiated and developed has been provided in the literature ${ }^{4-6}$. Here we briefly mention the overall science focus of AstroSat.

Electromagnetic emission observed across a large range of wavelengths enables us to extract signatures of radiative processes at the source. Often interactions of high-energy charged particles with matter and radiation give rise to photon emission across large bands of the spectrum. Key questions addressed by AstroSat include identifying locations and processes involved in the acceleration of particles to high energy, and the energy distribution and temporal behaviour of emitted radiation. Other environmental parameters like the presence of magnetic fields, though not directly imaged by AstroSat, can be extracted from the satellite observations. Details of accretion of matter onto compact objects like neutron stars and black holes by mechanisms not always known to us, are revealed through simultaneous multi-wavelength studies of AstroSat. High-resolution UV imaging with the UV imaging telescope (UVIT) reveals sites of formation of new stars, and permits quantification of star formation rates in our galaxy and external galaxies. The choice of different filters on UVIT also provides coarse spectral classification capability to derive ages, identify stellar groups and classify sources. The presence of scanning X-ray instrument on AstroSat provides an important ability to detect sources that exhibit large intensity variations.

\section{Science instruments}

To achieve these objectives, the following instruments were carefully selected and realized to cover a wide range 


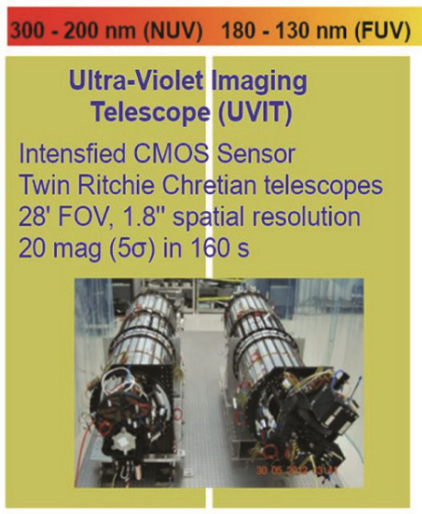

$0.3-8 \mathrm{keV}(\mathrm{SXT}) \quad 2.5-10 \mathrm{keV}(\mathrm{SSM}) \quad 3-100 \mathrm{keV}$ (LAXPC)

$25-150 \mathrm{keV}$ (CZTI)

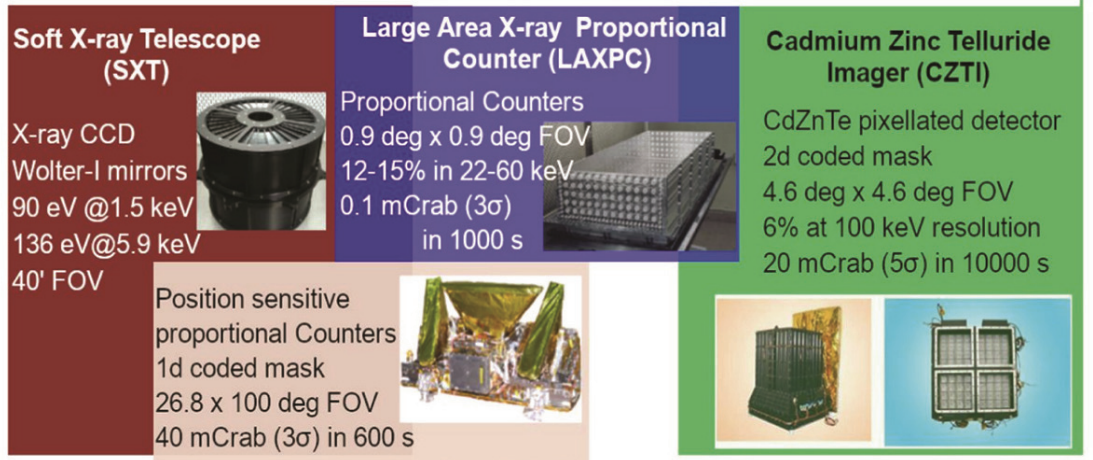

Scanning Sky Monitor (SSM)

Figure 1. Summary of five scientific instruments.

of spectral bands. Figure 1 summarizes the five science instruments on-board AstroSat, with major characteristics.

\section{Payload realization - a collaborative effort}

The AstroSat project is a fine example of an integrated effort not only by Indian agencies and institutions, but also by international agencies and universities for realizing a major astronomy mission. For the first time all the payloads, with the exception of scanning sky monitor (SSM), were completely designed, realized, tested, qualified, delivered and used by institutions other than ISRO. The national institutions include Tata Institute of Fundamental Research (TIFR), Mumbai; Indian Institute of Astrophysics (IIA), Bengaluru; Inter University Centre for Astronomy and Astrophysics (IUCAA), Pune; Raman Research Institute (RRI), Bengaluru and Physical Research Laboratory (PRL), Ahmedabad, along with international collaborations with the Canadian Space Agency (CSA), and University of Leicester, UK. Several Centres of ISRO have also been involved in development and testing of various subsystems of payloads, in addition to providing the spacecraft and launch vehicle.

\section{AstroSat project, mission and operations}

To achieve the scientific objectives, it was decided to configure a satellite in the $1500 \mathrm{~kg}$ class, and place it in an orbit of low background induced by South Atlantic Anomaly (SAA), with the capability to observe celestial sources in different portions of the sky. The SAA is a region where there is an increased concentration of energetic charged particles due to an anomaly of the inner Van Allen belts reaching closest to Earth. Many X-ray detectors also detect charged particles which have to be subtracted as background. In order to minimize the effect of increased background due to SAA in the X-ray detec- tors, a low inclination orbit is preferred. AstroSat was launched on 28 September 2015, with a lift-off mass of $1515 \mathrm{~kg}$ using the PSLV (Polar Satellite Launch Vehicle) C-30 launch vehicle, into a $650 \mathrm{~km}$ orbit, inclined at an angle of $6^{\circ}$ to the equator. This is the first near-equatorial launch for the PSLV, one more of its varied capacity. AstroSat had the highest payload to $\mathrm{S} / \mathrm{C}$ mass ratio of $>50 \%$ amongst the Indian satellites flown till then.

Some of the technical challenges in realizing payloads and spacecraft are the following:

- Indigenous development of UV mirrors to achieve a 28 arcmin field of view (FOV), and overall angular resolution of 1.8 arcsec, about three times better than the GALEX satellite.

- Indigenous development of gold-coated foils for soft X-ray optics, precision machining for the placement and alignment of 40 co-axial shells consisting of 320 mirrors.

- High-pressure, large-area, gas-filled proportional counters with effective area $>6000 \mathrm{~cm}^{2}$, energy measurement up to $80 \mathrm{keV}$, and a temporal resolution of $10 \mu \mathrm{s}$. Water-jet cutting system for fabricating the collimators.

- Development of position-sensitive gas-filled detectors and rotation mechanism for SSM.

- Qualification of commercial Cadmium Zinc telluride detectors for space astronomy.

- Capability for large on-board data storage with read/ write capability.

- Very stringent control on contamination of UVIT optics and other on-board systems.

- Setting up of a specialized M. G. K. Menon laboratory to assemble and test UV optics.

- Pre-launch inter-payload alignment and in-space measurement of alignments using stellar sources.

- S/C manoeuvring with avoidance of the Sun, and S/C pointing and stability.

- Simulation of background radiation, its estimate and modelling for the different detectors. 
Details of post-launch performance of the different payloads, and developments for the spacecraft are available in the literature ${ }^{7-19}$.

\section{AstroSat as an observatory}

AstroSat is ISRO's first science mission operated as a proposal-driven space observatory. The initial six months after the launch were used for testing and performance verification and tuning/optimization of payloads.

As an observatory open to Indian and international users, proposals with good scientific justification and technical feasibility to observe sources are solicited at frequent intervals. The AstroSat Proposal Processing System (APPS) is a web-based portal designed by IUCAA, Pune, and hosted and administered by the ISRO Space Science Data Centre (ISSDC), Bengaluru for submission and evaluation of proposals. The call for proposals, also termed as announcement of opportunity (AO) is typically issued in February/March of each year for an annual observation cycle starting from October. In addition, proposals are also submitted as target of opportunity (TOO) and calibration (Cal) proposals.

The submitted proposals are reviewed and approved through the APPS utility, by the AstroSat Time Allocation Committee (ATAC) with the help of technical feasibility assessment provided by the AstroSat Technical Committee (ATC). ATC considers payload operation constraints like avoidance of bright objects, constraints on source location with respect to the Sun, Moon, etc. and source detectability in various instruments. Based on these approvals, the mission and operations teams at the U.R. Rao Satellite Centre, and ISRO Telemetry Tracking and Command Network (ISTRAC) make the necessary list of targets to be observed, taking into consideration Sun avoidance, RAM angle constraint, data readout capability and thermal constraints, and finally generate command files ${ }^{20}$.

The UV mirrors and detectors are extremely sensitive to radiation from bright objects. It is necessary to avoid radiation from the Sun. To avert this, the satellite pointing is always maintained away from the Sun, which is called as Sun avoidance angle. The RAM angle is defined as the angle between the velocity vector and pointing axis and at zero degree RAM angle, the satellite will be ploughing through the particles (particularly atomic oxygen) in the orbit which can degrade the performance of the UV mirrors. To avoid this, the RAM angle is always maintained at more than $12^{\circ}$. Certain pointing directions are also avoided, if the expected temperature on some of the payload subsytems is beyond the acceptable range. The data generated by the payloads are stored in an on-board memory and downloaded when the satellite is visible to the ground. However, there are few restrictions when the antenna cannot be pointed to the Earth. Hence these durations are also avoided for observing the target stars. The scheduling of targets is done collectively involving various Centres of ISRO and considering proposal requirements. Payload and spacecraft operations and health monitoring are carried out at ISTRAC.

Raw data are received at ISSDC, and the final level-2 products are generated by the respective Payload Operation Centres (POCs) from the partially processed data provided by ISSDC. The final data are disseminated to the respective proposal Principal Investigators (PIs) by ISSDC. The data of observations under AO proposals have a lock-in period of 12 months with the proposal PIs, after which they are made available to the public as archival data. Transient sources which exhibit sudden outbursts, and/or sources that exhibit a change of state can be proposed for observations at a short notice as TOO. These proposals go through the same procedure of approvals by a TOO committee at a faster turn-around time. The TOO data do not have any lock-in period and are released simultaneously to the public and the proposer. The scientific validity of data of each instrument is checked at the respective POCs, which are run by one of the collaborating institutions for that payload using standard automated software. Both proprietary data (during lock-in) and archival data are hosted at 'https:// astrobrowse.issdc.gov.in' maintained by ISSDC.

Calibration proposals are also submitted by instrument teams for regular observations of sources meant for calibration of the instruments. The AstroSat calibration database (CALDB) is available at the AstroSat Science Support Cell (ASSC; http://astrosat-ssc.iucaa.in) hosted by IUCAA, Pune. ASSC is supported by ISRO. Software required for the analysis of AstroSat data is also available at ASSC. In addition to maintaining a suite of tools for extracting selected scientific products along with data analysis recipes, ASSC also provides assistance to proposers and conducts workshops for training users for proposal preparation and data analysis. Development of necessary tools for operating AstroSat as an observatoryclass satellite has been done in India itself for a space mission. Suitably modified versions of these tools are to be used for future space science missions.

\section{Summary of observations in five years}

Five years of operation of AstroSat has resulted in close to 150 refereed articles and more than 1500 circulars, conference proceedings and other non-refereed publications. AstroSat has also significantly contributed to 15 theses till now.

Currently, AstroSat has a total of 1483 registered users (AstroSat users) from 48 countries around the globe. Figure 2 shows the global distribution of these users. For clarity, only the countries with more than ten users are shown in the figure. About half of the AstroSat user base 
is from India. Thirty-two countries with individual number of AstroSat users less than ten are grouped together under 'Others'.

\section{Observation statistics}

AstroSat has observed a total of 944 unique fields over the last five years. Figure 3 shows the distribution of these pointings. Although the four scientific payloads on-board AstroSat point to the same direction providing simultaneous observations, the PI has the option of configuring one of the four payloads as the primary instrument depending on the science. Figure 3 also shows the distribution of AstroSat observations over the past years with different payloads as primary science instruments. From the figure it is evident that time utilized for UVIT observations is higher because it can only observe during the eclipse part of the orbit, and hence requires longer stare times to achieve the same observation time. Deep surveys also require longer stare time.

\section{Publications}

AstroSat data have resulted in more than 1500 publications till August 2020. Figure 4 shows the year-wise number of publications depicting increase in the utilization of AstroSat data with time. Only articles published in refereed journals, Astronomer's telegram and Gamma-ray Circular Network (GCN) circulars are plotted in the figure. For clarity, all the publications before 2015 are grouped together. Since the number of GCN circulars far exceeds the other publications, GCN number is scaled by 3. Some selected results are discussed in detail later in the text. In addition to refereed articles, AstroSat has resulted in $15 \mathrm{Ph} . \mathrm{D}$. theses.

\section{Major/selected results from AstroSat}

\section{Deep field survey using UVIT}

The search for sources providing evidence of emission of Lyman continuum photons supporting the concept of the epoch of re-ionization in the early Universe, is one of the interesting problems in astronomy. In the GOODS South field, the object AUDFs01 has been selected from the data of Hubble Extreme Deep field. AUDFs01 was found to have a good $\mathrm{S} / \mathrm{N}$ in the UV bands of AstroSat, which also corresponded to a redshifted emission of Lyman continuum. The estimated escape fraction of UV photons from this galaxy is $>20 \%$ (ref. 21). This observation of UVIT on-board AstroSat is made possible due to the reduced background of the UVIT detectors combined with the long exposure time. This detection, at a redshift $z \sim 1.42$, is the first detection of a Lyman continuum- emitting galaxy in the hitherto unexplored range $0.4<z<2.5$ in redshift, often referred to as the 'redshift desert $^{, 22,23}$. With further analysis, many such sources are expected to be detected.

Deep survey of M31 or Andromeda galaxy with UVIT led to the first detection of young main-sequence stars in the bulge of M31 outside the nucleus ${ }^{24}$. Based on this survey, a UVIT point source catalogue with about 75,000 sources in NUV or FUV has been produced ${ }^{25}$.

\section{Multi-wavelength studies}

Blazars are active galactic nuclei (AGNs) which have jets beamed towards the observer. Observations using SXT and LAXPC of the blazar RGB J0710+591 indicate that the X-ray part of the spectral energy distribution is unusually curved and can be explained in terms of synchrotron emission from a non-thermal distribution of high-energy electrons with a declining energy density around the peak ${ }^{26}$. The UV part of the spectrum appears to be of a different emission component than the X-ray part.

$\mathrm{X}$-ray binaries consist of a compact object (neutron star or a black hole) accreting matter from a companion star. The matter is often accreted through the formation of an accretion disc around the compact object. Her X-1 is an $\mathrm{X}$-ray binary exhibiting multi-wavelength emission. The X-ray data of this binary exhibit a 35-day cycle with low and high state, in addition to the orbital phase variation.

FUV emission from this source using UVIT indicates a variability starting near orbital phase of 0.3 . FUV emission is estimated from two components ${ }^{27}$ : (a) due to disc emission and (b) due to emission from heated portion of the companion star HZ Her. The geometry of the system is modelled by the authors, including the FUV data which

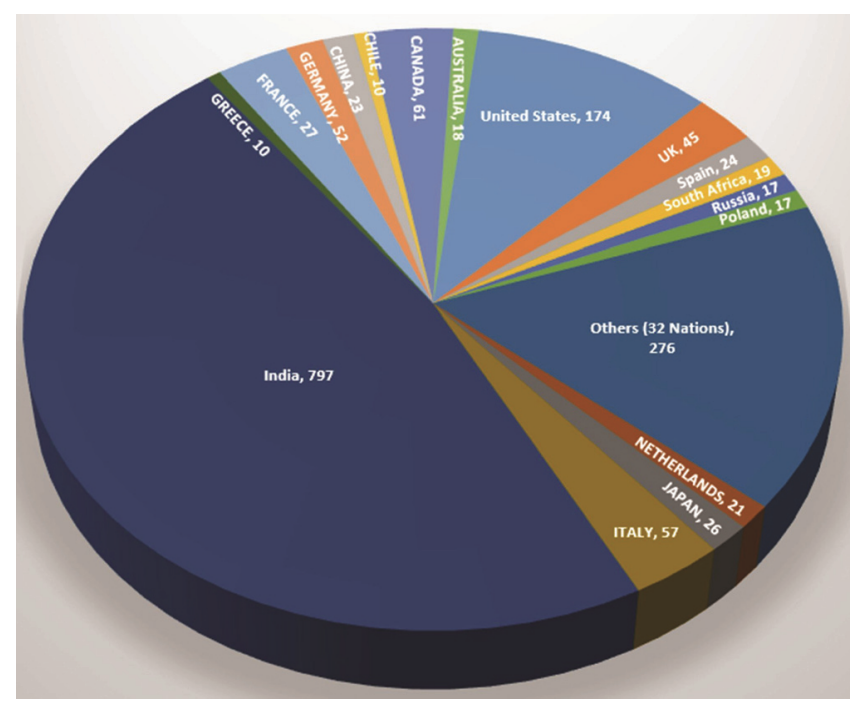

Figure 2. Global distribution of AstroSat users. For brevity, countries with less than ten users are grouped together. 

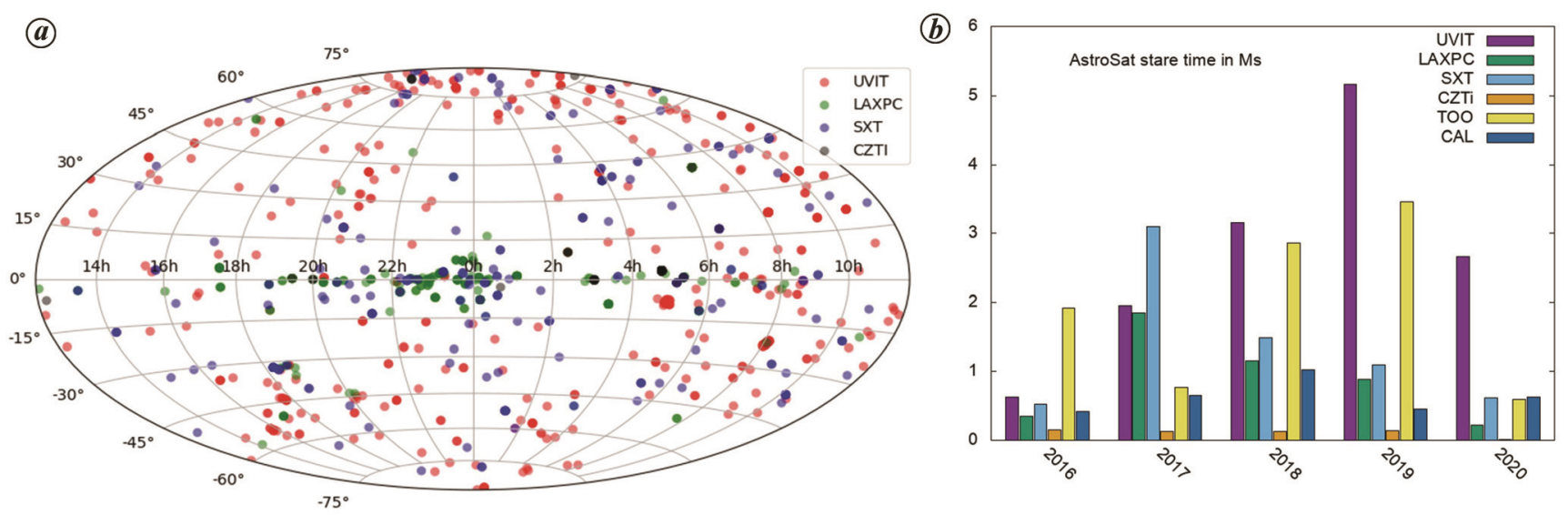

Figure 3. $\boldsymbol{a}$, Distribution of cosmic sources observed by AstroSat. $\boldsymbol{b}$, Stare time (Mega second) utilized for AstroSat observations with different payloads configured as main instruments as a function of time.

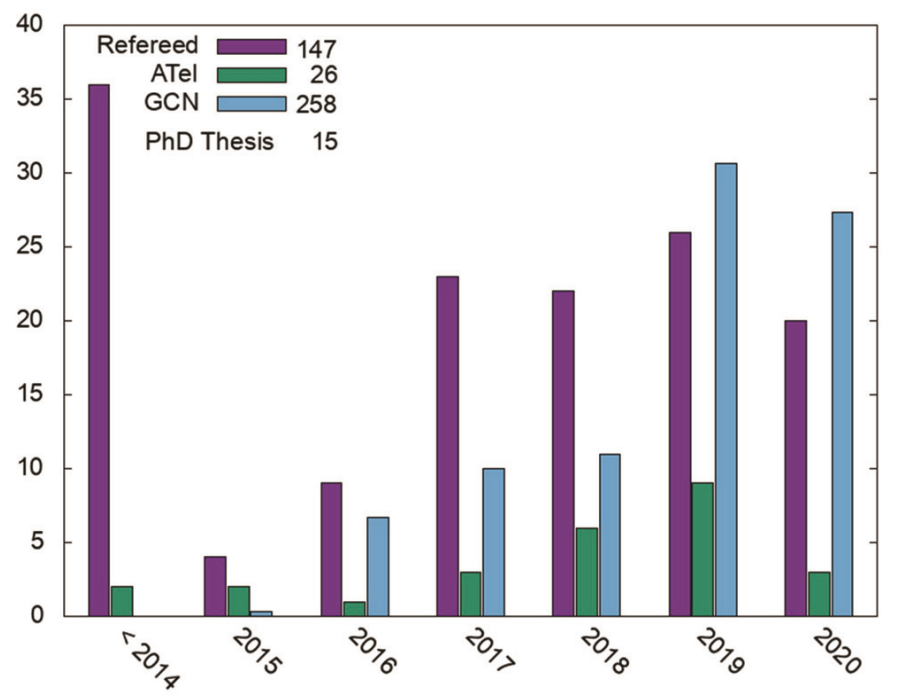

Figure 4. Distribution of AstroSat publications. The number of GCN circulars are scaled by 3 for clarity.

is commensurate with a tilted and twisted thin disk and does not require a thick inner disk.

The LAXPC data of Her X-1 exhibits a cyclotron line. This line feature has been studied for over three decades using data from various satellites, and was found to vary from 35 to $42 \mathrm{keV}$ with episodes of increase, decrease and constancy of the cyclotron energy. Using detailed modelling of earlier data and LAXPC data, it is confirmed that the cyclotron feature is dependent on both luminosity and time ${ }^{28}$. This dependency is explained by changes occurring in a mound formed by the accreted matter over the polar cap of the neutron star.

\section{Temporal variations}

Quasi-periodic oscillations (QPOs) occur close to the compact objects in X-ray binaries. As the name suggests, these oscillations are neither perfectly periodic nor are they always present in the data. Based on AstroSat data, QPOs have been detected around $\sim 1$ and $\sim 2 \mathrm{mHz}$ in $4 \mathrm{U}$ 0115+105 (ref. 29), at $20 \mathrm{mHz}$ in Cyg X-3 (ref. 30), at $47.7 \mathrm{mHz}$ with a weak oscillation at $109.4 \mathrm{mHz}$ in MAXI $\mathrm{J} 1820+070$ (ref. 31), at $2 \mathrm{~Hz}$ in MAXI J1535-57 (ref. 32) and at $5 \mathrm{~Hz}$ in $4 \mathrm{U} 1636-536$ (ref. 33). In the black hole binary GRS $1915+105$, the QPO varies in frequency from 2.55 to $6.55 \mathrm{~Hz}$ as the source flux almost doubles over a timescale of just $6 \mathrm{~h}$ (ref. 34).

High frequency QPOs have been observed at $\sim 50 \mathrm{~Hz}$ in neutron star binary GX5-1 (ref. 35), at $\sim 70 \mathrm{~Hz}$ in GRS $1915+105$ (ref. 36) and at $815 \mathrm{~Hz}$ evolving to $850 \mathrm{~Hz}$ in $4 \mathrm{U}$ 1728-34 (ref. 37). The 3-5 Hz QPOs detected in GRS $1915+105$ are identified to be due to the relativistically modified sound speed from the inner $\operatorname{disk}^{37}$. The spin parameter is estimated to be $0.973 \pm 0.002$. The highfrequency QPOs in GRS 1915+105 $(\sim 70 \mathrm{~Hz})$ also show 
variability and the hard photons $>10 \mathrm{keV}$ lag with respect to the soft photons ${ }^{38}$.

Variability studies of three blazars, viz. Mrk 421, PKS 2155-304 and 3C 273 have been conducted using the SXT on AstroSat and archived data of XMM-Newton satellite. A linear relationship is found between the rootmean-squared amplitude of variability at shorter timescales and mean flux level ${ }^{39}$.

\section{Black hole transients}

X-ray transients are sources which exhibit large variations in flux and spectral state changes over different timescales, and many physical parameters can be estimated during outbursts. Spectra of X-ray sources with both SXT and LAXPC provide not only a wide energy coverage from 0.3 to $80 \mathrm{keV}$, but also data with better $\mathrm{S} / \mathrm{N}$ in the soft band of the spectrum leading to improved modelling.

4U1630-47 was observed to be in the soft state during which the spectra can be fitted with a predominant disc spectrum. Usually the spin parameter of a black hole is estimated by fitting the gravitationally modified iron line. The X-ray continuum in a soft state using Chandra and AstroSat data has been used to estimate the spin parameter of $4 \mathrm{U} 1630-47$ to be 0.92 with over $99 \%$ confidence $^{40}$.

4U1630-47 was observed during both the 2016 and 2018 outbursts. With LAXPC and SXT data, the mass of the compact object has been estimated using three methods. These indicate a mass limit of 3-9 solar masses for the compact object ${ }^{41}$.

MAXI J1535-571 exhibits QPOS at $\sim 2 \mathrm{~Hz}$ (ref. 32), and the change in frequency is found to be correlated with power law index but not with luminosity ${ }^{42}$. The wideband spectrum appears to be fitted best with a model of spinning black hole, which yields an estimate of the mass of the black hole to be $10.39 \pm 0.61$ solar mass and the dimensionless spin parameter to be 0.67 (ref. 43).

\section{Cyclotron resonant scattering feature and bursts in neutron star binaries}

The detection of a $5 \mathrm{keV}$ cyclotron feature leading to a magnetic field estimate of $6 \times 10^{11} \mathrm{G}$ in SXP 15.3 makes it only the second pulsar in small Magellanic cloud with a confirmed magnetic field strength of the neutron star ${ }^{44}$.

Cyclotron features and their variation with pulse phase have also been observed in 4U 1538-522 at $22 \mathrm{keV}$ (ref. 45) and in 4U1907+09 at $18.5 \mathrm{keV}$ (ref. 46).

A few neutron-star X-ray binaries exhibit bursts. The bursts are considered to occur due to the thermonuclear burning of the accumulated accreted matter on the neutron star. One of the outstanding issues in modelling bursts has been whether the pre-burst persistent emission spectrum can be considered as non-changing and subtracted as background to estimate the burst spectrum. To assess this, the LAXPC data from 2016 outburst of $4 \mathrm{U}$ 1728-34 were analysed by dividing them into multiple bins. It was found that the persistent emission was enhanced and it could be due to the burst emission possibly increasing the disc emission by increasing the accretion rate $^{47}$.

A Be/X-ray binary GRO J2058+42 exhibited a strong outburst in April 2019 (ref. 48). This observation resulted in the detection of a QPO at $90 \mathrm{mHz}$ and its harmonics, indicative of formation of a disc. Spectral analysis led to the detection of three absorption features around $\sim 10,20$ and $38 \mathrm{keV}$. Magnetic field strength of $1.21 \times 10^{12} \mathrm{G}$ was estimated for the cyclotron feature at $10.81 \mathrm{keV}$.

\section{Stars, stellar clusters and galaxies}

The UVIT with its higher angular resolution combined with a large field of view is used to image and resolve stars in clusters and galaxies.

NGC 40, or the Bow-Tie Nebula, is a planetary nebula. UVIT detected a large halo of UV radiation in the FUV band surrounding the entire nebula. This halo is due to molecules energized by light from the central star ${ }^{49}$.

UV bright stars are identified in open cluster M67 with FUV observations using UVIT $^{50}$. Spectral energy distributions are estimated using the FUV data along with UV, optical and IR data from other observatories. Seven white dwarfs (WDs) have been identified, with four systems having extremely low mass WD as companions. This study indicates at least 12 sources which need further confirmation as WDs.

Proxima Centauri was observed in a coordinated campaign with Hubble Space Telescope and Chandra satellites. Using SXT, the quiescent and flare spectra were fitted with a three-temperature component model. It was found that during a flare, UV emission peak preceded the X-ray peak by $300-400 \mathrm{~s}$ possibly due to Neupert effect $^{51}$.

A globular cluster is a group of hundreds of thousands of stars tightly bound together by their own gravity. The globular cluster NGC 2808 has been observed in both NUV and FUV. The colour-magnitude diagrams reveal several stellar types in this cluster ${ }^{52}$. Stars in the red, blue and extreme horizontal branch, and post-asymptotic branch can be identified. The spatial extent of different groups seems to be indicative of different stellar populations.

NGC 2336, a spiral galaxy viewed nearly face-on was observed using UVIT. Seventy-two star-forming knots have been identified, with each knot having a size of about $400 \mathrm{pc}$ and the blue hotter knots being towards the centre $^{53}$. It is suggested that star-forming is driven by spiral density wave and is best studied in UV bands. 


\section{REVIEW ARTICLES}

Jelly-fish galaxies are those in which gas is stripped due to ram pressure. Observations in the NUV band of UVIT have confirmed eighty star-forming knots on the disk and tentacles of JO201 a jelly-fish galaxy in the Abell 85 cluster $^{54}$. The star-formation rate from FUV is $\sim 0.01$ to $2.07 \mathrm{M}_{\odot} \mathrm{yr}^{-1}$ and matches with $H_{\alpha}$ observations from ground-based observatories.

\section{Polarization studies}

Capability of polarization studies with the CZTI led to the detection and study of spin-phase dependence of polarization in $\mathrm{Crab}^{55}$. Recent spectro-polarimetric studies of the prompt emission of gamma-ray burst GRB160325A have been analysed to describe the two episodes of bursting activity ${ }^{56}$. CZTI observations have also been used to differentiate between the emission due to gravitational wave source GW 170104 and gamma-ray burst source GRB 170105A (ref. 57).

\section{Path ahead}

AstroSat has completed its design life of five years in September 2020. The fuel spent so far on the operations is negligible due to the excellent performance of the satellite. The spacecraft, barring unforeseen problems, is expected to be operational for many more years. Although some channels/units of some of the payloads have become unusable, it is proposed to maximize the use of AstroSat for best outcome in the coming years.

AstroSat has motivated many scientific teams to propose astronomy payloads/missions for the future. Among these are the X-ray polarization satellite, XpoSat and the solar observatory Aditya-L1, expected to be in orbit soon.

1. Marar, T. M. K. et al., The gamma-ray burst experiment onboard the SROSS-C satellite. $A \& A, 1994,283,698-704$.

2. Agrawal, P. C. et al., X-ray astronomy experiment on the Indian Satellite IRS-P3. JKASS, 1997, 29, 429.

3. Agrawal, P. C., ASTROSAT satellite. ASPC, 2001, 251, 512 513 .

4. Koteswara Rao, V. et al., The scientific objectives of the ASTROSAT mission of ISRO. Acta Astron., 2009, 65(1-2), 6-17.

5. Singh, K. P. et al., AstroSat mission. SPIE, 2014, 9144, 1.

6. Agrawal P. C., AstroSat: from inception to realisation and launch. J. Ap. A, 2017, 38, 27

7. Agrawal, P. C. et al., Large area X-ray proportional counter (LAXPC) instrument on AstroSat and some preliminary results from its performance in the orbit. J. Ap. A, 2017,38, 30

8. Antia, H. M., et al., Calibration of the large area X-ray proportional counter (LAXPC) instrument on board AstroSat. ApJS, 2017, 231, 10.

9. Bhalerao, V. et al., The cadmium zinc telluride imager on AstroSat. J. Ap. A, 2017, 38, 31.

10. Navalgund, K. H. et al., AstroSat - configuration and realization. J. Ap. A, 2017, 38, 34.

11. Ramadevi, M. C. et al., Early in-orbit performance of scanning sky monitor onboard AstroSat. J. Ap. A, 2017, 38, 32 .
12. Ramadevi, M. C. et al., Scanning sky monitor (SSM) onboard AstroSat. Exp Astron, 2017, 44, 11-23.

13. Rao, A. R. et al., Cadmium-zinc-telluride imager on-board AstroSat: a multi-faceted hard X-ray instrument. Curr. Sci., 2017, 113(4), 595-598.

14. Rao, A. R. et al., Charged particle monitor on the Astrosat mission. J. Ap. A, 2017, 38, 33.

15. Seetha, S. and Megala, Overview of the AstroSat mission. Curr. Sci., 2017, 113, 579-582.

16. Singh, K. P., et al., Proc. SPIE, in Space Telescopes and Instrumentation 2016: Ultraviolet to Gamma Ray. 9905, p. 99051E.

17. Singh, K. P. et al., Soft X-ray focusing telescope aboard AstroSat design, characteristics and performance. J. Ap. A, 2017, 38, 29.

18. Tandon, S. N. et al., In-orbit performance of UVIT and first results. J. Ap. A, 2017, 38, 28.

19. Tandon, S. N. et al., In-orbit calibrations of the ultraviolet imaging telescope. Astron. J., 2017, 154, 128-141.

20. Pandiyan, R. et al., Planning and scheduling of payloads of AstroSat during initial and normal phase observations. J. Ap. A, 2017, 38, 35.

21. Saha, K. et al., AstroSat detection of Lyman continuum emission from a $z=1.42$ galaxy. Nat. Astron., 2020, 164.

22. Steidel, C. C. et al., A survey of star-forming galaxies in the $1.4 \lesssim z \lesssim 2.5$ redshift desert: overview. ApJ, 2004, 604, 534550.

23. Renzini, A. and Daddi, E., Wandering in the redshift desert. Messenger, 2009, 137, 41-45.

24. Leahy, D. A., Bianchi, L. and Postma, J. E., ASTROSAT/UVIT survey of M31, first results UV-bright stars in the bulge. Astron. J., 2018, 156, 269-280.

25. Leahy, D. A. et al., AstroSat UVIT survey of M31: point-source catalog. APJSS, 2020, 247, 47-62.

26. Goswami, P. et al., Unravelling the unusually curved X-ray spectrum of RGB J0710+591 using AstroSat observations. MNRAS, 2020, 492, 796-803.

27. Leahy, D. A., Postma, J. and Chen, Y., AstroSat UVIT observations of Her X-1. ApJ, 2020, 889, 131-142.

28. Bala, S. et al., Time evolution of cyclotron line of Her X-1: a detailed statistical analysis including new AstroSat data. MNRAS, 2020, 497, 1029-1042.

29. Roy, J. et al., LAXPC/AstroSat study of $\sim 1$ and $\sim 2 \mathrm{mHz}$ quasiperiodic oscillations in the Be/X-ray binary $4 \mathrm{U} 0115+63$ during its 2015 outburst. ApJ, 2019, 872, 33-44.

30. Pahari, M. et al., X-ray timing analysis of Cyg X-3 using AstroSat/LAXPC: detection of milli-hertz quasi-periodic oscillations during the flaring hard X-ray state. ApJ, 2017, 849, 16-29.

31. Mudambi, S. et al., Unveiling the temporal properties of MAXI J1820+070 through AstroSat observations. ApJ. Lett., 2020, 889, L17-L24.

32. Sreehari, H. et al., AstroSat view of MAXI J1535-571: broad-band spectro-temporal features. MNRAS, 2019, 487, 928-941.

33. Beri, A. et al., Thermonuclear X-ray bursts in rapid succession in 4U 1636-536 with AstroSat-LAXPC. MNRAS, 2019, 482, 43974407.

34. Yadav, J. S. et al., Astrosat/LAXPC reveals the high-energy variability of GRS $1915+105$ in the X class. ApJ, 2016, 833, 2736.

35. Bhulla, Y. et al., AstroSat observation of GX 5-1: spectral and timing evolution. $R A A, 2019,19,114-136$.

36. Belloni, T. M. et al., A variable-frequency HFQPO in GRS $1915+105$ as observed with AstroSat. MNRAS, 2019, 489, 10371043.

37. Verdhan, C. J. et al., AstroSat/LAXPC detection of millisecond phenomena in 4U 1728-34. ApJ, 2017, 841, 41-46.

38. Misra, R. et al., Identification of QPO frequency of GRS $1915+105$ as the relativistic dynamic frequency of a truncated accretion disk. ApJ. Lett., 2020, 889, L36-L42. 
39. Bhattacharyya, S. et al., Blazar variability: a study of nonstationarity and the flux-rms relation. ApJ, 2020, 897, 25-35.

40. Pahari, M. et al., AstroSat and Chandra view of the high soft state of $4 \mathrm{U}$ 1630-47 (4U 1630-472): evidence of the disk wind and a rapidly spinning black hole. ApJ, 2018, 867, 86-96.

41. Baby, B. E. et al., AstroSat and MAXI view of the black hole binary 4U 1630-472 during 2016 and 2018 outbursts. MNRAS, 2020, 497, 1197-1211

42. Bhargava, Y. et al., Spectro-timing analysis of MAXI J1535-571 using AstroSat. MNRAS, 2019, 488, 720-727.

43. Sridhar, N. et al., Broad-band reflection spectroscopy of MAXI J1535-571 using AstroSat: estimation of black hole mass and spin. MNRAS, 2019, 487, 4221-4229.

44. Maitra, C. et al., Detection of a cyclotron line in SXP 15.3 during its 2017 outburst. MNRAS, 2018, 480, L136.

45. Varun, Maitra, C. et al., Probing the cyclotron line characteristics of 4U 1538-522 using AstroSat-LAXPC. MNRAS, 2019, 484, L1L6.

46. Varun, Pradhan, P. et al., Pulse phase variation of the cyclotron line in HMXB 4U 1907+09 with AstroSat LAXPC. ApJ, 2019, 880, 61-68.

47. Bhattacharyya, S., Yadav, J. S. and Sridhar, N., Effects of thermonuclear X-ray bursts on non-burst emissions in the soft state of $4 \mathrm{U}$ 1728-34. ApJ, 2018, 860, 88.

48. Mukerjee, K., Antia, H. M. and Katoch, T., AstroSat observations of GRO J2058+42 during the 2019 outburst. ApJ, 2020, 897, 7391.

49. Rao, N. K. et al., Planetary nebulae with UVIT around the Bow Tie nebula (NGC 40). $A \& A, 2018, \mathbf{6 0 9}, \mathrm{L} 1-\mathrm{L} 4$.

50. Jadhav, V., Sindhu, N. and Annapurni S., UVIT Open Cluster Study. II. Detection of extremely low mass white dwarfs and postmass transfer binaries in M67. ApJ, 2019, 886, 13-35.
51. Lalitha, S. et al., Proxima Centauri-the nearest planet host observed simultaneously with AstroStat, Chandra, and HST. MNRAS, 2020, 498, 3658-3663.

52. Jain, R., Vig, S. and Ghosh, S. K., Investigation of the globular cluster NGC 2808 with the ultra-violet imaging telescope. MNRAS, 2019, 485, 2877-2888.

53. Rahna, P. T. et al., A study of the star-forming regions in the spiral galaxy NGC 2336 using the Ultraviolet Imaging Telescope (UVIT). MNRAS, 2018, 481, 1212.

54. George, K. et al., UVIT view of ram-pressure stripping in action: star formation in the stripped gas of the GASP jellyfish galaxy JO201 in Abell 85. MNRAS, 2018, 479, 4126-4135.

55. Vadawale, S. et al., Phase resolved X-ray polarimetry of the Crab pulsar with the AstroSat CZT Imager. Nature Astron., 2018, 2, $50-55$.

56. Sharma, V. et al., Spectropolarimetric analysis of prompt emission of GRB 160325A: jet with evolving environment of internal shocks. MNRAS, 2020, 493, 5218-5232.

57. Bhalerao, V. et al., A tale of two transients: GW 170104 and GRB 170105A. ApJ, 2017, 845, 152-162.

ACKNOWLEDGEMENTS. We thank the reviewers for their useful comments and Dr K. Kasturirangan for initiating the AstroSat project as the then Chairman of ISRO. We also thank Shri A. S. Kiran Kumar (ISRO, HQ) who motivated us to write this article and Dr P. Sreekumar (ISRO, HQ) for guiding us through it.

Received 11 December 2020; re-revised accepted 31 May 2021

doi: $10.18520 / \mathrm{cs} / \mathrm{v} 121 / \mathrm{i} 2 / 214-221$ 